\title{
AN URN MODEL ARISING FROM ALL-OPTICAL NETWORKS
}

\author{
JOHN A. MORRISON,* Bell Laboratories
}

\begin{abstract}
An occupancy model that has arisen in the investigation of randomized distributed schedules in all-optical networks is considered. The model consists of $B$ initially empty urns, and at stage $j$ of the process $d_{j} \leq B$ balls are placed in distinct urns with uniform probability. Let $M_{i}(j)$ denote the number of urns containing $i$ balls at the end of stage $j$. An explicit expression for the joint factorial moments of $M_{0}(j)$ and $M_{1}(j)$ is obtained. A multivariate generating function for the joint factorial moments of $M_{i}(j), 0 \leq i \leq I$, is derived (where $I$ is a positive integer). Finally, the case in which the $d_{j}, j \geq 1$, are independent, identically distributed random variables is investigated.
\end{abstract}

Keywords: Generating function; joint factorial moment; occupancy model; optical network; randomized schedule

2000 Mathematics Subject Classification: Primary 05A15

Secondary 60K30; 90B15

\section{Introduction}

In a recent paper, Morrison et al. [3] investigated randomized distributed (RAD) schedules in all-optical networks. A schedule consists of $B$ time slots of the same duration. Both sourcebased (S-RAD) and destination-based (D-RAD) schedules were considered. In S-RAD, sources acting independently of each other assign time slots for transmitting to destinations. In D-RAD, destinations acting independently of each other assign time slots for receiving transmissions from sources. In each case there is a possibility of conflict. In the case of S-RAD, transmissions may arrive simultaneously at a destination from more than one source. In the case of D-RAD, a source may be given times to transmit to more than one destination.

Mathematically, S-RAD and D-RAD may each be formulated as an occupancy model with $B$ urns. At stage $j$ of the process, $d_{j}$ balls are placed in distinct urns with uniform probability. In D-RAD,$d_{j}$ corresponds to the number of time slots assigned to a given source by destination $j$. Let $D_{N}=\sum_{j=1}^{N} d_{j} \leq B$, where $N$ is the number of destinations, and let $M_{0}(N)$ denote the number of empty urns. Then the number of conflicts is $D_{N}-B+M_{0}(N)$, since the source may transmit to one of the destinations in a time slot which has been assigned by more than one destination. In S-RAD, $d_{j}$ corresponds to the number of time slots assigned by source $j$ for transmission to a given destination, and $N$ corresponds to the number of sources. Let $M_{1}(N)$ denote the number of urns containing just one ball at the end of stage $N$. Then the number of conflicts is $D_{N}-M_{1}(N)$, because of arrivals in a time slot at the destination from more than one source.

Explicit expressions were derived in [3] for the first- and second-order moments of $M_{0}(N)$ and $M_{1}(N)$. These were used to evaluate the mean and variance of the blocking probabilities.

Received 26 January 2005; revision received 14 September 2006.

* Postal address: Bell Laboratories, Lucent Technologies, 600 Mountain Avenue, Murray Hill, NJ 07974, USA.

Email address: johnmorrison@lucent.com 
The second-order moments were derived from equations for the factorial and joint moments. Higher-order moments of $M_{0}(N)$ and $M_{1}(N)$ provide further insight into the S-RAD and $\mathrm{D}-\mathrm{RAD}$ processes. Consequently, special consideration is given in this paper to these moments. The approach adopted here facilitates the investigation of the more general problem discussed below, and leads to the classical result [1] if $d_{j}=1, j \geq 1$.

If $d_{j}=d$, where $j \geq 1$ and $d$ is a positive integer, then the urn model corresponds to allocation of particles by complexes [2, Chapter VI], and if $d=1$ it corresponds to the classical problem [1]. In this paper, we consider the more general case and investigate the joint factorial moments of all orders of the occupancies $M_{i}(j)$, the number of urns containing $i$ balls at the end of stage $j$, for $0 \leq i \leq I$ (where $I$ is a positive integer). The urn problem is formulated in Section 2. The joint factorial moments are investigated in Section 3, and a recursion on $j$ is derived. An explicit expression for the joint factorial moments of $M_{0}(j)$ and $M_{1}(j)$ is derived in Section 4. It is shown that the recursion on $j$ for a multivariate exponential generating function for the joint factorial moments of $M_{i}(j), 0 \leq i \leq I$, for $I \geq 2$ leads to partial derivatives with respect to the variables. An explicit alternative generating function for the joint factorial moments is derived in Section 5. The case in which the $d_{j}, j \geq 1$, are independent, identically distributed random variables is investigated in Section 6.

\section{Formulation}

We consider an urn model [3] that consists of $B$ urns which are initially empty. At stage $j$ of the process, $d_{j} \leq B$ balls are placed in distinct urns with uniform probability. Let $M_{i}(j)$ denote the number of urns containing $i$ balls at the end of stage $j$, and let

$$
P_{j}\left(s_{0}, s_{1}, \ldots, s_{I}\right)=\operatorname{Pr}\left\{M_{i}(j)=s_{i}, 0 \leq i \leq I\right\} .
$$

Then $M_{i}(j)=0$ if $i>j$ and

$$
P_{j}\left(s_{0}, s_{1}, \ldots, s_{I}\right)=0 \quad \text { if } \sum_{i=0}^{I} s_{i}>B .
$$

The binomial coefficient $\left(\begin{array}{l}n \\ m\end{array}\right)$ is taken to be 0 when $m>n$ or $n<0$. Then $P_{j}\left(s_{0}, s_{1}, \ldots, s_{I}\right)$ satisfies the following recurrence.

Theorem 1. For the above urn model,

$$
\begin{aligned}
& P_{j}\left(s_{0}, s_{1}, \ldots, s_{I}\right) \\
& =\sum_{\sum_{i=0}^{I+1} a_{i}=d_{j}}\left(\begin{array}{c}
s_{0}+a_{0} \\
a_{0}
\end{array}\right) \prod_{i=1}^{I}\left(\begin{array}{c}
s_{i}+a_{i}-a_{i-1} \\
a_{i}
\end{array}\right)\left(\begin{array}{c}
B-\sum_{i=0}^{I} s_{i}-a_{I} \\
a_{I+1}
\end{array}\right)\left(\begin{array}{c}
B \\
d_{j}
\end{array}\right)^{-1} \\
& \quad \times P_{j-1}\left(s_{0}+a_{0}, s_{1}+a_{1}-a_{0}, \ldots, s_{I}+a_{I}-a_{I-1}\right), \quad j \geq 1,
\end{aligned}
$$

where $a_{i}, 0 \leq i \leq I+1$, are nonnegative integers. In terms of the Kronecker delta, the initial condition is

$$
P_{0}\left(s_{0}, s_{1}, \ldots, s_{I}\right)=\delta_{s_{0}, B} \prod_{i=1}^{I} \delta_{s_{i}, 0} .
$$


Proof. The proof of (2) follows from conditioning on how state $\left(s_{0}, s_{1}, \ldots, s_{I}\right)$ may be reached at stage $j$ from stage $j-1$, and counting the number of ways this may occur. Thus, state $\left(s_{0}, s_{1}, \ldots, s_{I}\right)$ is reached at stage $j$ if $a_{0}$ balls are placed in $s_{0}+a_{0}$ empty urns, $a_{i}$ balls are placed in $s_{i}+a_{i}-a_{i-1}$ urns containing $i$ balls, $1 \leq i \leq I$, and the remaining $a_{I+1}=d_{j}-\sum_{i=0}^{I} a_{i}$ balls are placed in $B-\sum_{i=0}^{I} s_{i}-a_{I}$ urns containing more than $I$ balls at stage $j-1$. The number of ways that the $d_{j}$ balls may be placed in the $B$ urns is given by the binomial coefficient $\left(\begin{array}{c}B \\ d_{j}\end{array}\right)$.

\section{Joint factorial moments}

The $n$th descending factorial of $l$ is

$$
l^{(n)}=\frac{l !}{(l-n) !} .
$$

For $m_{i} \geq 0,0 \leq i \leq I$, we define $\boldsymbol{m}=\left(m_{0}, \ldots, m_{I}\right)$ and the joint factorial moments

$$
\begin{aligned}
\Psi_{j}(\boldsymbol{m}) & =\mathrm{E}\left\{\prod_{i=0}^{I}\left[M_{i}(j)\right]^{\left(m_{i}\right)}\right\} \\
& =\sum_{s_{0}=0}^{\infty} \cdots \sum_{s_{I}=0}^{\infty} \prod_{i=0}^{I} s_{i}^{\left(m_{i}\right)} P_{j}\left(s_{0}, \ldots, s_{I}\right) .
\end{aligned}
$$

We assume that $\sum_{i=0}^{I} m_{i} \leq B$, since $\prod_{i=0}^{I} s_{i}^{\left(m_{i}\right)}=0$ unless $m_{i} \leq s_{i}, 0 \leq i \leq I$, and $P_{j}\left(s_{0}, \ldots, s_{I}\right)=0$ unless $\sum_{i=0}^{B} s_{i} \leq B$, from (1). Also, $\Psi_{j}(\boldsymbol{m})=0$ unless $m_{i}=0$ for $i>j$, since $M_{i}(j)=0$ if $i>j$.

Lemma 1. It follows from Theorem 1 and (5) that

$$
\begin{aligned}
\left(\begin{array}{c}
B \\
d_{j}
\end{array}\right) \Psi_{j}(\boldsymbol{m})=\sum_{s_{0}=0}^{\infty} \cdots \sum_{s_{I}=0}^{\infty} & \sum_{\sum_{i=0}^{I+1} a_{i}=d_{j}}\left(s_{0}-a_{0}\right)^{\left(m_{0}\right)} \prod_{i=1}^{I}\left(s_{i}+a_{i-1}-a_{i}\right)^{\left(m_{i}\right)} \\
& \times \prod_{i=0}^{I}\left(\begin{array}{c}
s_{i} \\
a_{i}
\end{array}\right)\left(\begin{array}{c}
B-\sum_{i=0}^{I} s_{i} \\
a_{I+1}
\end{array}\right) P_{j-1}\left(s_{0}, \ldots, s_{I}\right), \quad j \geq 1,
\end{aligned}
$$

and

$$
\Psi_{0}(\boldsymbol{m})=B^{\left(m_{0}\right)} \prod_{i=1}^{I} \delta_{m_{i}, 0} .
$$

Proof. We make use of (2) in (5), and replace $s_{0}+a_{0}$ by $s_{0}$ and $s_{i}+a_{i}-a_{i-1}$ by $s_{i}$, $1 \leq i \leq I$. Since $\left(\begin{array}{l}s_{i} \\ a_{i}\end{array}\right)=0$ if $s_{i}<a_{i}$, the lower limits in the sums for the new $s_{i}, 0 \leq i \leq I$, may be taken to be 0 . The initial condition (7) follows from (3) and (5).

The goal is to express the right-hand side of (6) in terms of $\Psi_{j-1}(\cdot)$. We will make use of the following lemmas for this purpose.

Lemma 2. For $1 \leq i \leq I$,

$$
\left(s_{i}+a_{i-1}-a_{i}\right)^{\left(m_{i}\right)}=\sum_{k_{i}=0}^{m_{i}}\left(\begin{array}{c}
m_{i} \\
k_{i}
\end{array}\right) a_{i-1}^{\left(k_{i}\right)}\left(s_{i}-a_{i}\right)^{\left(m_{i}-k_{i}\right)} .
$$


Proof. The result follows directly from the identity

$$
(c+\gamma)^{(m)}=\left[\frac{\mathrm{d}^{m}}{\mathrm{~d} x^{m}}(1+x)^{c+\gamma}\right]_{x=0}=\left[\frac{\mathrm{d}^{m}}{\mathrm{~d} x^{m}}\left\{(1+x)^{c}(1+x)^{\gamma}\right\}\right]_{x=0}=\sum_{k=0}^{m}\left(\begin{array}{l}
m \\
k
\end{array}\right) c^{(k)} \gamma^{(m-k)} .
$$

The following identities follow directly from (4).

Lemma 3. For $s_{0} \geq a_{0}+m_{0}$ and $a_{0} \geq k_{1}$,

$$
a_{0}^{\left(k_{1}\right)}\left(s_{0}-a_{0}\right)^{\left(m_{0}\right)}\left(\begin{array}{c}
s_{0} \\
a_{0}
\end{array}\right)=s_{0}^{\left(m_{0}+k_{1}\right)}\left(\begin{array}{c}
s_{0}-m_{0}-k_{1} \\
a_{0}-k_{1}
\end{array}\right) ;
$$

for $s_{i} \geq a_{i}+m_{i}-k_{i}$ and $a_{i} \geq k_{i+1}$,

$$
a_{i}^{\left(k_{i+1}\right)}\left(s_{i}-a_{i}\right)^{\left(m_{i}-k_{i}\right)}\left(\begin{array}{c}
s_{i} \\
a_{i}
\end{array}\right)=s_{i}^{\left(m_{i}-k_{i}+k_{i+1}\right)}\left(\begin{array}{c}
s_{i}-m_{i}+k_{i}-k_{i+1} \\
a_{i}-k_{i+1}
\end{array}\right), \quad 1 \leq i \leq I-1 ;
$$

and, for $s_{I} \geq a_{I}+m_{I}-k_{I}$,

$$
\left(s_{I}-a_{I}\right)^{\left(m_{I}-k_{I}\right)}\left(\begin{array}{c}
s_{I} \\
a_{I}
\end{array}\right)=s_{I}^{\left(m_{I}-k_{I}\right)}\left(\begin{array}{c}
s_{I}-m_{I}+k_{I} \\
a_{I}
\end{array}\right) .
$$

With the help of Lemmas 2 and 3 we may evaluate the sum over the $a_{i}, \sum_{i=0}^{I+1} a_{i}=d_{j}$, in (6), as follows.

Lemma 4. Let

$$
m=\sum_{i=0}^{I} m_{i}, \quad k=\sum_{i=1}^{I} k_{i}
$$

Then

$$
\begin{aligned}
& \sum_{\sum_{i=0}^{I+1} a_{i}=d_{j}}\left(\begin{array}{c}
s_{0}-m_{0}-k_{1} \\
a_{0}-k_{1}
\end{array}\right) \prod_{i=1}^{I-1}\left(\begin{array}{c}
s_{i}-m_{i}+k_{i}-k_{i+1} \\
a_{i}-k_{i+1}
\end{array}\right)\left(\begin{array}{c}
s_{I}-m_{I}+k_{I} \\
a_{I}
\end{array}\right)\left(\begin{array}{c}
B-\sum_{i=0}^{I} s_{i} \\
a_{I+1}
\end{array}\right) \\
& \quad=\left(\begin{array}{c}
B-m \\
d_{j}-k
\end{array}\right) .
\end{aligned}
$$

Proof. We rewrite the sum as

$$
\sum_{i=0}^{I-1}\left(a_{i}-k_{i+1}\right)+a_{I}+a_{I+1}=d_{j}-k
$$

and note that $a_{i}^{\left(k_{i+1}\right)}=0$ if $a_{i}<k_{i+1}$ and that $a_{i} \geq k_{i+1}, 0 \leq i \leq I-1, a_{i} \geq 0$, and $a_{I+1} \geq 0$ imply that $d_{j} \geq k$. We also note that

$$
B-m=\left(s_{0}-m_{0}-k_{1}\right)+\sum_{i=1}^{I-1}\left(s_{i}-m_{i}+k_{i}-k_{i+1}\right)+\left(s_{I}-m_{I}+k_{I}\right)+\left(B-\sum_{i=0}^{I} s_{i}\right) .
$$

The result in (9) follows by factoring $(1+x)^{B-m}$ according to (10), expanding in powers of $x$, and equating the coefficients of $x^{d_{j}-k}$. 
We note that the right-hand side of (9) does not depend on $s_{i}, 0 \leq i \leq I$. Hence, again with the help of Lemmas 2 and 3, we may express the resulting sums over the $s_{i}, 0 \leq i \leq I$, in (6) in terms of $\Psi_{j-1}(\cdot)$. It is convenient to define vectors $\boldsymbol{e}_{i}$ with $1 \mathrm{~s}$ in the $i$ th places and $0 \mathrm{~s}$ elsewhere, and write

$$
\boldsymbol{m}=\sum_{i=0}^{I} m_{i} \boldsymbol{e}_{i}, \quad\left(\boldsymbol{e}_{i}\right)_{n}=\delta_{i n} .
$$

We also make use of the identity

$$
B^{(m)}\left(\begin{array}{c}
B-m \\
d_{j}-k
\end{array}\right)=d_{j}^{(k)}\left(B-d_{j}\right)^{(m-k)}\left(\begin{array}{c}
B \\
d_{j}
\end{array}\right),
$$

to obtain the following recurrence.

Theorem 2. For $0 \leq m \leq B$, the joint factorial moments satisfy

$$
\begin{aligned}
B^{(m)} \Psi_{j}\left(\sum_{i=0}^{I} m_{i} \boldsymbol{e}_{i}\right)= & \sum_{k_{1}=0}^{m_{1}} \cdots \sum_{k_{I}=0}^{m_{I}} \prod_{i=1}^{I}\left(\begin{array}{c}
m_{i} \\
k_{i}
\end{array}\right) d_{j}^{(k)}\left(B-d_{j}\right)^{(m-k)} \\
& \times \Psi_{j-1}\left(m_{0} \boldsymbol{e}_{0}+\sum_{i=1}^{I}\left[\left(m_{i}-k_{i}\right) \boldsymbol{e}_{i}+k_{i} \boldsymbol{e}_{i-1}\right]\right), \quad j \geq 1,
\end{aligned}
$$

where $m$ and $k$ are as given by (8).

Saniee (personal communication (2004)) has derived (11) in the case $m_{i}=\delta_{i I}$, by means of indicator functions which denote whether or not urns contain $i$ balls at the end of stage $j$. Presumably this approach may be extended to obtain the general result in (11).

\section{Exponential generating functions}

Because of its relevance to all-optical networks, we first consider

$$
\Psi_{j}(m-n, n)=\mathrm{E}\left\{\left[M_{0}(j)\right]^{(m-n)}\left[M_{1}(j)\right]^{(n)}\right\}, \quad 0 \leq n \leq m .
$$

From (11), for $0 \leq m \leq B$ we obtain

$$
B^{(m)} \Psi_{j}(m-n, n)=\sum_{k=0}^{n}\left(\begin{array}{l}
n \\
k
\end{array}\right) d_{j}^{(k)}\left(B-d_{j}\right)^{(m-k)} \Psi_{j-1}(m-n+k, n-k), \quad j \geq 1 .
$$

We now extend the range of $n$ in (13) to $\infty$, and introduce the exponential generating function

$$
g_{j}(m, x)=\sum_{n=0}^{\infty} \Psi_{j}(m-n, n) \frac{x^{n}}{n !} .
$$

We then obtain the following explicit result.

Theorem 3. For $0 \leq m \leq B$,

$$
g_{j}(m, x)=B^{(m)} \prod_{l=1}^{j} \frac{1}{B^{(m)}} \sum_{k=0}^{\infty} d_{l}^{(k)}\left(B-d_{l}\right)^{(m-k)} \frac{x^{k}}{k !}, \quad j \geq 1 .
$$


Proof. It follows from (13) and (14) that

$$
\begin{aligned}
B^{(m)} g_{j}(m, x) & =\sum_{k=0}^{\infty} d_{j}^{(k)}\left(B-d_{j}\right)^{(m-k)} \frac{x^{k}}{k !} \sum_{n=k}^{\infty} \Psi_{j-1}(m-n+k, n-k) \frac{x^{n-k}}{(n-k) !} \\
& =\sum_{k=0}^{\infty} d_{j}^{(k)}\left(B-d_{j}\right)^{(m-k)} \frac{x^{k}}{k !} g_{j-1}(m, x), \quad j \geq 1 .
\end{aligned}
$$

However, from (7) and (14) we have $g_{0}(m, x)=B^{(m)}$, and (15) follows.

As expected, the order of the stages $l, 1 \leq l \leq j$, in (15) is irrelevant. We note that the sum in (15) is finite, since $d_{l}^{(k)}=0$ if $k>d_{l}$. From (12) and (14), E $\left\{\left[M_{0}(j)\right]^{(m-n)}\left[M_{1}(j)\right]^{(n)}\right\}$ is the coefficient of $x^{n} / n$ ! in $g_{j}(m, x)$. In particular, from (15),

$$
\mathrm{E}\left\{\left[M_{0}(j)\right]^{(m)}\right\}=B^{(m)} \prod_{l=1}^{j} \frac{\left(B-d_{l}\right)^{(m)}}{B^{(m)}}, \quad 0 \leq m \leq B,
$$

and

$$
\mathrm{E}\left\{\left[M_{0}(j)\right]^{(m-1)} M_{1}(j)\right\}=\sum_{i=1}^{j} d_{i}\left(B-d_{i}\right)^{(m-1)} \prod_{\substack{l=1 \\ l \neq i}}^{j} \frac{\left(B-d_{l}\right)^{(m)}}{B^{(m)}}, \quad 1 \leq m \leq B,
$$

where an empty product is taken to be equal to 1 . The results for $m=1$ and $m=2$ were derived in [3], and an alternative derivation, using indicator functions, has been obtained by Saniee (personal communication (2004)), without the use of recursions on $j$. Also,

$$
\begin{aligned}
& \mathrm{E}\left\{\left[M_{0}(j)\right]^{(m-2)}\left[M_{1}(j)\right]^{(2)}\right\} \\
& =\frac{2}{B^{(m)}} \sum_{n=1}^{j} \sum_{i=1}^{n-1} d_{i}\left(B-d_{i}\right)^{(m-1)} d_{n}\left(B-d_{n}\right)^{(m-1)} \prod_{\substack{l=1 \\
l \neq i \\
l \neq n}}^{j} \frac{\left(B-d_{l}\right)^{(m)}}{B^{(m)}} \\
& \quad+\sum_{i=1}^{j} d_{i}^{(2)}\left(B-d_{i}\right)^{(m-2)} \prod_{\substack{l=1 \\
l \neq i}}^{j} \frac{\left(B-d_{l}\right)^{(m)}}{B^{(m)}}, \quad 2 \leq m \leq B,
\end{aligned}
$$

where an empty sum is taken to be equal to 0 . The result for $m=2$ was derived in [3]. Generally,

$$
\begin{array}{r}
\mathrm{E}\left\{\left[M_{0}(j)\right]^{(m-n)}\left[M_{1}(j)\right]^{(n)}\right\}=n ! B^{(m)} \sum_{\sum_{l=1}^{j} n_{l}=n} \prod_{l=1}^{j} \frac{d_{l}^{\left(n_{l}\right)}\left(B-d_{l}\right)^{\left(m-n_{l}\right)}}{B^{(m)} n_{l} !}, \\
0 \leq n \leq m \leq B .
\end{array}
$$

For $I \geq 2$, we extend the range of $m_{i}, 1 \leq i \leq I$, in (11) to $\infty$, and introduce the multivariate exponential generating function

$$
g_{j}(m ; \boldsymbol{x})=\sum_{m_{1}=0}^{\infty} \cdots \sum_{m_{I}=0}^{\infty} \Psi_{j}\left(m \boldsymbol{e}_{0}+\sum_{i=1}^{I} m_{i}\left(\boldsymbol{e}_{i}-\boldsymbol{e}_{0}\right)\right) \prod_{i=1}^{I} \frac{x_{i}^{m_{i}}}{m_{i} !},
$$

where $\boldsymbol{x}=\left(x_{1}, \ldots, x_{I}\right)$. We then obtain the following result. 
Theorem 4. For $0 \leq m \leq B$, let

$$
D(k ; \boldsymbol{x})=\sum_{\sum_{i=1}^{I} k_{i}=k} \prod_{i=1}^{I} \frac{x_{i}^{k_{i}}}{k_{i} !} \frac{\partial^{k_{2}+\cdots+k_{I}}}{\partial x_{1}^{k_{2}} \cdots \partial x_{I-1}^{k_{I}}}
$$

and

$$
\Phi_{j}(m ; \boldsymbol{x})=\frac{1}{B^{(m)}} \sum_{k=0}^{\infty} d_{j}^{(k)}\left(B-d_{j}\right)^{(m-k)} D(k ; \boldsymbol{x}) .
$$

Then

$$
g_{j}(m ; \boldsymbol{x})=\Phi_{j}(m ; \boldsymbol{x})\left[g_{j-1}(m ; \boldsymbol{x})\right], \quad j \geq 1,
$$

and

$$
g_{0}(m ; \boldsymbol{x})=B^{(m)} .
$$

Proof. From (11) and (19), if we reverse the order of summation over $m_{i}$ and $k_{i}$, and set $n_{i}=m_{i}-k_{i}, 1 \leq i \leq I$, then we obtain

$$
B^{(m)} g_{j}(m ; \boldsymbol{x})=\sum_{k_{1}=0}^{\infty} \cdots \sum_{k_{I}=0}^{\infty} d_{j}^{(k)}\left(B-d_{j}\right)^{(m-k)} \prod_{i=1}^{I} \frac{x^{k_{i}}}{k_{i} !} h_{j-1}(m ; \boldsymbol{x}, \boldsymbol{k}),
$$

where $\boldsymbol{k}=\left(k_{1}, \ldots, k_{I}\right), k=\sum_{i=1}^{I} k_{i}$, and

$$
h_{j-1}(m ; \boldsymbol{x} ; \boldsymbol{k})=\sum_{n_{1}=0}^{\infty} \cdots \sum_{n_{I}=0}^{\infty} \Psi_{j-1}\left(m \boldsymbol{e}_{0}+\sum_{i=1}^{I} n_{i}\left(\boldsymbol{e}_{i}-\boldsymbol{e}_{0}\right)+\sum_{i=2}^{I} k_{i}\left(\boldsymbol{e}_{i-1}-\boldsymbol{e}_{0}\right)\right) \prod_{i=1}^{I} \frac{x_{i}^{n_{i}}}{n_{i} !} .
$$

However, from (19), we find that

$$
\begin{aligned}
& \frac{\partial^{k_{2}+\cdots+k_{I}}}{\partial x_{1}^{k_{2}} \cdots \partial x_{I-1}^{k_{I}}} g_{j-1}(m ; \boldsymbol{x}) \\
& \quad=\sum_{m_{1}=k_{2}}^{\infty} \cdots \sum_{m_{I-1}=k_{I}}^{\infty} \sum_{m_{I}=0}^{\infty} \Psi_{j-1}\left(m \boldsymbol{e}_{0}+\sum_{i=1}^{I} m_{i}\left(\boldsymbol{e}_{i}-\boldsymbol{e}_{0}\right)\right) \prod_{i=1}^{I-1} \frac{x_{i}^{m_{i}-k_{i+1}}}{\left(m_{i}-k_{i+1}\right) !} \frac{x_{I}^{m_{I}}}{m_{I} !} .
\end{aligned}
$$

If we let $n_{i}=m_{i}-k_{i+1}, 1 \leq i \leq I-1$, and $n_{I}=m_{I}$, then we find that the right-hand side of (24) is equal to $h_{j-1}(m ; \boldsymbol{x} ; \boldsymbol{k})$, and (22) follows. The initial condition (23) follows from (7) and (19).

We note that the sum in (21) is finite, since $d_{j}^{(k)}=0$ if $k>d_{j}$. The order of the stages $l$, $1 \leq l \leq j$, should be irrelevant, and we establish this with the help of the following result, proved in Appendix A.

Theorem 5. The partial differential operator $D(k ; \boldsymbol{x})$ defined by $(20)$ is commutative and, hence, from (21),

$$
\Phi_{j}(m ; \boldsymbol{x}) \Phi_{l}(m ; \boldsymbol{x})=\Phi_{l}(m ; \boldsymbol{x}) \Phi_{j}(m ; \boldsymbol{x}) .
$$

We obtain the following result from (22) and (23). 
Corollary 1. The exponential generating function is given by

$$
g_{j}(m ; \boldsymbol{x})=B^{(m)} \prod_{l=1}^{j} \Phi_{l}(m ; \boldsymbol{x})[1] .
$$

In particular,

$$
g_{1}(m ; \boldsymbol{x})=\sum_{k=0}^{\infty} d_{1}^{(k)}\left(B-d_{1}\right)^{(m-k)} \frac{x_{1}^{k}}{k !}
$$

and

$$
\begin{gathered}
g_{2}(m ; \boldsymbol{x})=\frac{1}{B^{(m)}} \sum_{n=0}^{\infty} \sum_{k=0}^{\infty} d_{2}^{(n)}\left(B-d_{2}\right)^{(m-n)} d_{1}^{(k)}\left(B-d_{1}\right)^{(m-k)} \\
\times \sum_{n_{2}=0}^{\min (k, n)} \frac{x_{1}^{k+n-2 n_{2}} x_{2}^{n_{2}}}{\left(k-n_{2}\right) !\left(n-n_{2}\right) ! n_{2} !} .
\end{gathered}
$$

If $d_{j}=1, j \geq 1$, then, from (20)-(22),

$$
B g_{j}(m ; \boldsymbol{x})=\left(B-m+x_{1}+\sum_{i=2}^{I} x_{i} \frac{\partial}{\partial x_{i-1}}\right) g_{j-1}(m ; \boldsymbol{x}), \quad j \geq 1 .
$$

We introduce the generating function

$$
G(m ; \boldsymbol{x} ; z)=\sum_{j=0}^{\infty} \frac{(B z)^{j}}{j !} g_{j}(m ; \boldsymbol{x}) .
$$

From (23) and (26), we have

$$
\frac{\partial G}{\partial z}=\left(B-m+x_{1}\right) G+\sum_{i=2}^{I} x_{i} \frac{\partial G}{\partial x_{i-1}}, \quad G(m ; \boldsymbol{x} ; 0)=B^{(m)} .
$$

The solution to this may be obtained by the method of characteristics, and it is readily verified that

$$
G(m ; \boldsymbol{x} ; z)=B^{(m)} \mathrm{e}^{(B-m) z} \exp \left(\sum_{i=1}^{I} \frac{x_{i} z^{i}}{i !}\right) .
$$

Hence, with $M=\sum_{i=1}^{I} i m_{i}$,

$$
g_{j}(m ; \boldsymbol{x})=j ! B^{(m)} \sum_{m_{1}=0}^{\infty} \cdots \sum_{m_{I}=0}^{\infty} \frac{(1-m / B)^{j-M}}{B^{M}(j-M) !} \prod_{i=1}^{I} \frac{x_{i}^{m_{i}}}{(i !)^{m_{i}} m_{i} !} .
$$

From (5) and (19) we obtain the classical result [1]

$$
\mathrm{E}\left\{\prod_{i=0}^{I}\left[M_{i}(j)\right]^{\left(m_{i}\right)}\right\}=\frac{j ! B^{(m)}(1-m / B)^{j-M}}{B^{M}(j-M) ! \prod_{i=1}^{I}(i !)^{m_{i}}}, \quad m=\sum_{i=0}^{I} m_{i} .
$$

We adopt a different approach in the next section. 


\section{Alternative generating function}

To analyze the joint factorial moments in general, we first state an equivalent form of Theorem 2.

Theorem 6. For $0 \leq m \leq B$ and positive integers $l_{p}$,

$$
\begin{aligned}
B^{(m)} \Psi_{j}\left(\sum_{p=1}^{m} \boldsymbol{e}_{l_{p}}\right)= & \left(B-d_{j}\right)^{(m)} \Psi_{j-1}\left(\sum_{p=1}^{m} \boldsymbol{e}_{l_{p}}\right) \\
& +d_{j}\left(B-d_{j}\right)^{(m-1)} \sum_{p=1}^{m} 1_{\left\{l_{p}>0\right\}} \Psi_{j-1}\left(\boldsymbol{e}_{l_{p}-1}+\sum_{\substack{q=1 \\
q \neq p}}^{m} \boldsymbol{e}_{l_{q}}\right) \\
& +\sum_{k=2}^{m-1} d_{j}^{(k)}\left(B-d_{j}\right)^{(m-k)} \sum_{p(1)<\cdots<p(k)} 1_{\left\{l_{p(n)>0}: 1 \leq n \leq k\right\}} \\
& \times \Psi_{j-1}\left(\sum_{n=1}^{k} \boldsymbol{e}_{l_{p(n)}-1}+\sum_{n=k+1}^{m} \boldsymbol{e}_{l_{p(n)}}\right) \\
& +d_{j}^{(m)} 1_{\left\{l_{p}>0: 1 \leq p \leq m\right\}} \Psi_{j-1}\left(\sum_{p=1}^{n} \boldsymbol{e}_{l_{p-1}}\right), \quad j \geq 1,
\end{aligned}
$$

where $1 \leq p(n) \leq m$ for $1 \leq n \leq k$ and $1_{\{\cdot\}}$ denotes the indicator function.

Proof. Let

$$
\sum_{i=0}^{I} m_{i} \boldsymbol{e}_{i}=\sum_{p=1}^{m} \boldsymbol{e}_{l_{p}}
$$

so that $l_{p}=i$ for $m_{i}$ values of $p, 0 \leq i \leq I$. The number of terms in (28) corresponding to $k=\sum_{i=1}^{I} k_{i}$ is

$$
\left(\begin{array}{c}
m-m_{0} \\
k
\end{array}\right)=\sum_{\sum_{i=1}^{I} k_{i}=k} \prod_{i=1}^{I}\left(\begin{array}{c}
m_{i} \\
k_{i}
\end{array}\right)
$$

However, for each set $\left(k_{1}, \ldots, k_{I}\right), \prod_{i=1}^{I}\left(\begin{array}{c}m_{i} \\ k_{i}\end{array}\right)$ counts the number of arguments of $\Psi_{j-1}$ in (28) which are equal to $m_{0} \boldsymbol{e}_{0}+\sum_{i=1}^{I}\left[\left(m_{i}-k_{i}\right) \boldsymbol{e}_{i}+k_{i} \boldsymbol{e}_{i-1}\right]$. The theorem then follows from (11).

We again let $\boldsymbol{x}=\left(x_{1}, \ldots, x_{m}\right)$, and define the symmetric functions

$$
S_{0}(\boldsymbol{x})=1, \quad S_{1}(\boldsymbol{x})=\sum_{p=1}^{m} x_{p}, \quad S_{k}(\boldsymbol{x})=\sum_{p(1)<\cdots<p(k)} \prod_{n=1}^{k} x_{p(n)}, \quad 2 \leq k \leq m .
$$

In particular,

$$
S_{m}(\boldsymbol{x})=\prod_{p=1}^{m} x_{p}
$$


We also define the multivariate generating function

$$
f_{j}(m ; \boldsymbol{x})=\sum_{l_{1}=0}^{\infty} \cdots \sum_{l_{m}=0}^{\infty} \Psi_{j}\left(\sum_{p=1}^{m} \boldsymbol{e}_{l_{p}}\right) \prod_{p=1}^{m}\left(x_{p}\right)^{l_{p}} .
$$

We then obtain the following explicit result.

Theorem 7. For $j \geq 1$,

$$
f_{j}(m ; \boldsymbol{x})=B^{(m)} \prod_{l=1}^{j} \frac{1}{B^{(m)}} \sum_{k=0}^{m} d_{l}^{(k)}\left(B-d_{l}\right)^{(m-k)} S_{k}(\boldsymbol{x}) .
$$

Proof. It follows from (28), (30), and (31) that

$$
B^{(m)} f_{j}(m ; \boldsymbol{x})=\sum_{k=0}^{m} d_{j}^{(k)}\left(B-d_{j}\right)^{(m-k)} S_{k}(\boldsymbol{x}) f_{j-1}(m ; \boldsymbol{x}) .
$$

However, from (7) and (31),

$$
f_{0}(m ; \boldsymbol{x})=B^{(m)},
$$

and (32) follows.

Corresponding to (29), we take

$$
l_{p}=i, \quad \sum_{r=0}^{i-1} m_{r}+1 \leq p \leq \sum_{r=0}^{i} m_{r}, 0 \leq i \leq I,
$$

where empty sums are equal to 0 . Then, from (5) and (31), $\mathrm{E}\left\{\prod_{i=0}^{I}\left[M_{i}(j)\right]^{\left(m_{i}\right)}\right\}$ is the coefficient of

$$
\prod_{i=0}^{I}\left(\prod_{p=\sum_{r=0}^{i-1} m_{r}+1}^{\sum_{r=0}^{i} m_{r}} x_{p}\right)^{i}
$$

in $f_{j}(m ; \boldsymbol{x})$, where empty products are equal to 1 . In particular, $\mathrm{E}\left\{\left[M_{i}(j)\right]^{(m)}\right\}$ is the coefficient of $\left(\prod_{p=1}^{m} x_{p}\right)^{i}$ in $f_{j}(m ; \boldsymbol{x})$. If $d_{j}=1, j \geq 1$, then, from (30) and (32),

$$
\begin{aligned}
f_{j}(m ; \boldsymbol{x}) & =B^{(m)}\left[\left(1-\frac{m}{B}\right)+\frac{1}{B} \sum_{p=1}^{m} x_{p}\right]^{j} \\
& =B^{(m)} \sum_{k=0}^{j} \frac{j !}{(j-k) !}\left(1-\frac{m}{B}\right)^{j-k} \frac{1}{B^{k}} \sum_{\sum_{p=1}^{m} n_{p}=k} \prod_{p=1}^{m} \frac{x_{p}^{n_{p}}}{n_{p} !},
\end{aligned}
$$

which again leads to (27) (the classical result).

Generally, for $0 \leq n \leq m, \mathrm{E}\left\{\left[M_{0}(j)\right]^{(m-n)}\left[M_{1}(j)\right]^{(n)}\right\}$ is the coefficient of $\prod_{p=m-n+1}^{m} x_{p}$ in $f_{j}(m ; \boldsymbol{x})$ and, hence, because of the symmetry in $\boldsymbol{x}$, it is also the coefficient of $\prod_{p=1}^{n} x_{p}$. Thus,

$$
\mathrm{E}\left\{\left[M_{0}(j)\right]^{(m-n)}\left[M_{1}(j)\right]^{(n)}\right\}=\left.\frac{\partial^{n} f_{j}(m ; \boldsymbol{x})}{\partial x_{1} \cdots \partial x_{n}}\right|_{\boldsymbol{x}=\mathbf{0}} .
$$


However, from (30) we obtain

$$
S_{k}(\mathbf{0})=\delta_{k 0},\left.\quad \frac{\partial S_{k}(\boldsymbol{x})}{\partial x_{q}}\right|_{\boldsymbol{x}=\mathbf{0}}=\delta_{k 1}
$$

and

$$
\left.\frac{\partial^{r} S_{k}(\boldsymbol{x})}{\partial x_{q(1)} \cdots \partial x_{q(r)}}\right|_{\boldsymbol{x}=\mathbf{0}}=\delta_{k r}, \quad q(1)<\cdots<q(r), 2 \leq r \leq m .
$$

The result in (18) follows from (32) and (33)-(35).

We now consider the first- and second-order factorial moments of $M_{i}(j)$. First, from (30) and (32),

$$
\sum_{i=0}^{j} \mathrm{E}\left\{M_{i}(j)\right\} x^{i}=f_{j}(1 ; x)=B \prod_{l=1}^{j}\left(1-\frac{d_{l}}{B}+\frac{d_{l}}{B} x\right)
$$

It follows that

$$
\mathrm{E}\left\{M_{0}(j)\right\}=B \prod_{l=1}^{j}\left(1-\frac{d_{l}}{B}\right), \quad \mathrm{E}\left\{M_{1}(j)\right\}=\sum_{n=1}^{j} d_{n} \prod_{\substack{l=1 \\ l \neq n}}\left(1-\frac{d_{l}}{B}\right)
$$

corresponding to $m=1$ in (16) and (17), and

$$
\mathrm{E}\left\{M_{i}(j)\right\}=B \sum_{l(1)<\cdots<l(i)} \prod_{r=1}^{i} \frac{d_{l(r)}}{B} \prod_{\substack{l=1 \\ l \neq l(r), 1 \leq r \leq i}}^{j}\left(1-\frac{d_{l}}{B}\right), \quad 2 \leq i \leq j .
$$

Saniee (personal communication (2004)) derived the results in (36) and (37) directly by means of indicator functions.

Next, $\mathrm{E}\left\{M_{k}(j)\left[M_{i}(j)-\delta_{i k}\right]\right\}$ is the coefficient of $x^{i} y^{k}$ in

$$
\begin{aligned}
f_{j}(2 ;(x, y)) & =B(B-1) \prod_{l=1}^{j}\left\{\left(1-\frac{d_{l}}{B}\right)\left[1-\frac{d_{l}}{(B-1)}+\frac{d_{l}}{(B-1)}(x+y)\right]+\frac{d_{l}\left(d_{l}-1\right)}{B(B-1)} x y\right\} \\
& =\sum_{r+s=0}^{j} A_{r, s}(x y)^{r}(x+y)^{s} \\
& =\sum_{r+s=0}^{j} A_{r, s}(x y)^{r} \sum_{p=0}^{s}\left(\begin{array}{l}
s \\
p
\end{array}\right) x^{p} y^{s-p}
\end{aligned}
$$

where the coefficients $A_{r, s}$ may be written down explicitly. Hence,

$$
\mathrm{E}\left\{M_{k}(j)\left[M_{i}(j)-\delta_{i k}\right]\right\}=\sum_{p=i-k}^{\min (i, j-k)}\left(\begin{array}{c}
2 p+k-i \\
p
\end{array}\right) A_{i-p, 2 p+k-i}, \quad 0 \leq k \leq i \leq j .
$$




\section{Random number of balls}

So far we have assumed that at stage $j$ of the process a prescribed number, $d_{j} \leq B$, of balls are placed in distinct urns. We now consider the case when the $d_{j}, j \geq 1$, are independent random variables with common distribution

$$
\operatorname{Pr}\left\{d_{j}=r\right\}=P(r), \quad \sum_{r=1}^{B} P(r)=1 .
$$

Now,

$$
\mathrm{E}\left\{\prod_{i=0}^{I}\left[M_{i}(j)\right]^{\left(m_{i}\right)}\right\}=\sum_{d_{1}=1}^{B} \cdots \sum_{d_{j}=1}^{B} \mathrm{E}\left\{\prod_{i=0}^{I}\left[M_{i}(j)\right]^{\left(m_{i}\right)} \mid d_{l}, 1 \leq l \leq j\right\} \prod_{l=1}^{j} P\left(d_{l}\right) .
$$

The corresponding generating function is, for $j \geq 1$,

$$
\begin{aligned}
F_{j}(m ; \boldsymbol{x}) & =\sum_{d_{1}=1}^{B} \cdots \sum_{d_{j}=1}^{B} f_{j}(m ; \boldsymbol{x}) \prod_{l=1}^{j} P\left(d_{l}\right) \\
& =B^{(m)}\left\{\frac{1}{B^{(m)}} \sum_{k=0}^{m} \mathrm{E}\left\{d_{l}^{(k)}\left(B-d_{l}\right)^{(m-k)}\right\} S_{k}(\boldsymbol{x})\right\}^{j},
\end{aligned}
$$

from (32).

Analogously, from (15), for $0 \leq n \leq m$ and $j \geq 1, \mathrm{E}\left\{\left[M_{0}(j)\right]^{(m-n)}\left[M_{1}(j)\right]^{(n)}\right\}$ is the coefficient of $x^{n} / n$ ! in

$$
\begin{aligned}
G_{j}(m, x) & =\sum_{d_{1}=1}^{B} \cdots \sum_{d_{j}=1}^{B} g_{j}(m, x) \prod_{l=1}^{j} P\left(d_{l}\right) \\
& =B^{(m)}\left\{\frac{1}{B^{(m)}} \sum_{k=0}^{\infty} \mathrm{E}\left\{d_{l}^{(k)}\left(B-d_{l}\right)^{(m-k)}\right\} \frac{x^{k}}{k !}\right\}^{j}
\end{aligned}
$$

We let

$$
c_{k m}=\frac{1}{B^{(m)}} \mathrm{E}\left\{d_{l}^{(k)}\left(B-d_{l}\right)^{(m-k)}\right\}=\frac{1}{B^{(m)}} \sum_{r=1}^{B}\left[r^{(k)}(B-r)^{(m-k)}\right] P(r) .
$$

Then, from (38) and (39),

$$
F_{j}(m ; \boldsymbol{x})=B^{(m)}\left[\sum_{k=0}^{m} c_{k m} S_{k}(\boldsymbol{x})\right]^{j}, \quad G_{j}(m, x)=B^{(m)}\left(\sum_{k=0}^{\infty} c_{k m} \frac{x^{k}}{k !}\right)^{j} .
$$

In the nonrandom case, corresponding to $P(r)=\delta_{r d}$,

$$
d_{j}=d, \quad j \geq 1, \quad c_{k m}=d^{(k)} \frac{(B-d)^{(m-k)}}{B^{(m)}} .
$$


As a simple example,

$$
F_{j}(1 ; x)=B\left(c_{01}+c_{11} x\right)^{j}=B \sum_{i=0}^{j}\left(\begin{array}{l}
j \\
i
\end{array}\right)\left(c_{01}\right)^{j-i}\left(c_{11} x\right)^{i} .
$$

Hence,

$$
\mathrm{E}\left\{M_{i}(j)\right\}=B\left(\begin{array}{l}
j \\
i
\end{array}\right)\left(c_{01}\right)^{j-i}\left(c_{11}\right)^{i}, \quad 0 \leq i \leq j .
$$

If $d_{j}=d, j \geq 1$, then, from (40), $c_{01}=1-d / B$ and $c_{11}=d / B$, and (41) agrees with the result of $[2$, Chapter VI].

Next,

$$
F_{j}(2 ;(x, y))=B(B-1)\left[c_{02}+c_{12}(x+y)+c_{22} x y\right]^{j} .
$$

The coefficient of $y^{l}$ in $F_{j}(2 ;(x, y))$ is

$$
\begin{aligned}
B(B-1)\left(\begin{array}{l}
j \\
l
\end{array}\right)\left(c_{12}+c_{22} x\right)^{l}\left(c_{02}+c_{12} x\right)^{j-l} \\
\quad=B(B-1) j ! \sum_{n=0}^{l} \frac{\left(c_{22} x\right)^{n}\left(c_{12}\right)^{l-n}}{n !(l-n) !} \sum_{q=0}^{j-l} \frac{\left(c_{12} x\right)^{q}\left(c_{02}\right)^{j-l-q}}{q !(j-l-q) !} .
\end{aligned}
$$

Hence, for $0 \leq l \leq i \leq j$, the coefficient of $x^{i} y^{l}$ in $F_{j}(2 ;(x, y))$ is

$$
\mathrm{E}\left\{M_{l}(j)\left[M_{i}(j)-\delta_{i l}\right]\right\}=B(B-1) j ! \sum_{n=0}^{l} \frac{\left(c_{22}\right)^{n}\left(c_{12}\right)^{l+i-2 n}\left(c_{02}\right)^{j-l-i+n}}{n !(l-n) !(i-n) !(j-l-i+n) !} .
$$

If $d_{j}=d, j \geq 1$, then, from (40),

$$
c_{02}=\frac{(B-d)(B-d-1)}{B(B-1)}, \quad c_{12}=\frac{d(B-d)}{B(B-1)}, \quad c_{22}=\frac{d(d-1)}{B(B-1)},
$$

and, for $0 \leq l \leq i \leq j$,

$$
\begin{aligned}
& \mathrm{E}\left\{M_{l}(j)\left[M_{i}(j)-\delta_{i l}\right]\right\} \\
& \quad=\frac{j ! d^{i}}{[B(B-1)]^{j-1}} \sum_{n=0}^{l} \frac{(d-1)^{n} d^{l-n}(B-d)^{j-n}(B-d-1)^{j-l-i+n}}{n !(l-n) !(i-n) !(j-l-i+n) !} .
\end{aligned}
$$

\section{Appendix A.}

Here we prove Theorem 5. From (20), we obtain

$$
D(k ; \boldsymbol{x}) D(n ; \boldsymbol{x})=\sum_{\sum_{i=1}^{I} k_{i}=k} \sum_{\sum_{i=1}^{I} n_{i}=n} \theta(\boldsymbol{k}, \boldsymbol{n} ; \boldsymbol{x}),
$$

where

$$
\theta(\boldsymbol{k}, \boldsymbol{n} ; \boldsymbol{x})=\sum_{r_{2}=0}^{k_{2}} \cdots \sum_{r_{I}=0}^{k_{I}} \prod_{i=1}^{I-1} \frac{x_{i}^{k_{i}+n_{i}-r_{i+1}}}{r_{i+1} !} \frac{x_{I}^{k_{I}+n_{I}}}{\phi} \prod_{i=2}^{I} \frac{\partial^{k_{i}+n_{i}-r_{i}}}{\partial x_{i-1}^{k_{i}+n_{i}-r_{i}}}
$$


and

$$
\begin{aligned}
\phi & =k_{1} ! \prod_{i=1}^{I-1}\left(k_{i+1}-r_{i+1}\right) !\left(n_{i}-r_{i+1}\right) ! n_{I} ! \\
& =\left(k-\sum_{i=2}^{I} k_{i}\right) ! \prod_{i=2}^{I}\left(k_{i}-r_{i}\right) !\left(n-\sum_{i=2}^{I} n_{i}-r_{2}\right) ! \prod_{i=2}^{I-1}\left(n_{i}-r_{i+1}\right) ! n_{I} !
\end{aligned}
$$

where an empty product is equal to 1 . We let $k_{i}+n_{i}=s_{i}$ and consider prescribed values of $k$, $n$, and $r_{i}$ and $s_{i}, 2 \leq i \leq I$. Then

$$
\begin{aligned}
\phi= & \left(k-\sum_{i=2}^{I} k_{i}\right) ! \prod_{i=2}^{I}\left(k_{i}-r_{i}\right) !\left[n+\sum_{i=2}^{I}\left(k_{i}-s_{i}\right)-r_{2}\right] ! \\
& \times \prod_{i=2}^{I-1}\left(s_{i}-r_{i+1}-k_{i}\right) !\left(s_{I}-k_{I}\right) !
\end{aligned}
$$

so $1 / \phi$ vanishes unless $r_{i} \leq s_{i}-r_{i+1}, 2 \leq i \leq I-1$, and $r_{I} \leq s_{I}$.

We first let $I=2$. If we replace $k_{2}$ by $s_{2}+r_{2}-k_{2}$ in the expression

$$
\sum_{k_{2}=r_{2}}^{s_{2}} \frac{1}{\phi}=\sum_{k_{2}=r_{2}}^{s_{2}}\left[\left(k-k_{2}\right) !\left(k_{2}-r_{2}\right) !\left(n+k_{2}-s_{2}-r_{2}\right) !\left(s_{2}-k_{2}\right) !\right]^{-1},
$$

we find that it is symmetric in $k$ and $n$. For $I \geq 3$, if we replace $k_{i}$ by $s_{i}+r_{i}-r_{i+1}-k_{i}$, $2 \leq i \leq I-1$, and $k_{I}$ by $s_{i}+r_{I}-k_{I}$ in the expression

$$
\sum_{k_{2}=r_{2}}^{s_{2}-r_{3}} \cdots \sum_{k_{I-1}=r_{I-1}}^{s_{I-1}-r_{I}} \sum_{k_{I}=r_{I}}^{s_{I}} \frac{1}{\phi}
$$

where $\phi$ is given by (44), we find that it is symmetric in $k$ and $n$. It follows from (42) and (43), by summing over $k_{i}+n_{i}=s_{i}, 2 \leq i \leq I$, and noting that $k_{1}+n_{1}=k+n-\sum_{i=2}^{I} s_{i}$, that

$$
D(k ; \boldsymbol{x}) D(n ; \boldsymbol{x})=D(n ; \boldsymbol{x}) D(k ; \boldsymbol{x}) .
$$

However, from (21),

$$
\Phi_{j}(m ; \boldsymbol{x}) \Phi_{l}(m ; \boldsymbol{x})=\frac{1}{\left[B^{(m)}\right]^{2}} \sum_{k=0}^{\infty} \sum_{n=0}^{\infty} d_{j}^{(k)}\left(B-d_{j}\right)^{(m-k)} d_{l}^{(n)}\left(B-d_{l}\right)^{(m-n)} D(k ; \boldsymbol{x}) D(n ; \boldsymbol{x}) .
$$

If we interchange $k$ and $n$ and use (45), we obtain (25).

\section{Acknowledgement}

The author is grateful to Iraj Saniee for bringing this problem to his attention and for giving an equivalent formulation for the joint distribution of the number of empty and single-ball urns. 


\section{References}

[1] Johnson, N. L. And Kotz, S. (1977). Urn Models and Their Application. An Approach to Modern Discrete Probability Theory. John Wiley, New York.

[2] Kolchin, V. F., Sevast’yanov, B. A. and Christiakov, V. P. (1978). Random Allocations. V. H. Winston, Washington, DC.

[3] Morrison, J. A., Saniee, I. And Widjaja, I. (2006). Design and performance of randomized network schedules for time-domain wavelength interleaved networks. Preprint. 